\title{
Zweiter bundesrätlicher Eingriff in den TARMED
}

\author{
Thomas Kesslera, Patrick Müller \\ axperte Abteilung Ambulante Versorgung und Tarife, FMH; ${ }^{\mathrm{b}}$ Leiter Abteilung Ambulante Versorgung und Tarife, $\mathrm{FMH}$
}

Der Bundesrat nimmt seine gesetzliche subsidiäre Kompetenz in Form eines zweiten Eingriffes in die national-einheitliche Tarifstruktur TARMED erneut wahr. Dieser zweite Eingriff führt zu zahlreichen und umfassenden Änderungen der Tarifstruktur TARMED, wovon sämtliche Ärztinnen und Ärzte direkt betroffen sind. Die definitive Verordnung mit sämtlichen Details liegt nun vor und tritt per 1.1.2018 in Kraft. Im folgenden Artikel werden dazu die wichtigsten Punkte aufgezeigt.

Zahlreiche Ärzteorganisationen und auch einzelne Ärzte haben die Gelegenheit sich zur dreimonatigen Vernehmlassung des zweiten subsidiären Eingriffs des Bundesrates in den TARMED genutzt und eine Stellungnahme zuhanden des BAG eingereicht. Die FMH hat am 20. Juni 2017 in Bern in einer Medienkonferenz zum zweiten Tarifeingriff Stellung genommen und hat die in enger Zusammenarbeit mit vielen Dach- und Fachgesellschaften erarbeitete Stellungnahme vorgestellt. Ein Auszug der Stellungnahme wurde in der Schweizerischen Ärztezeitung 28/29 vom 12.07.2017 publiziert und ist auf der Webseite der FMH zu finden. Das Departement Ambulante Versorgung und Tarife der FMH hat umfassende Analysen und Simulationen der Massnahmen des zweiten Tarifeingriffes gemeinsam mit den Fachgesellschaften und den kantonalen Ärztegesellschaften durchgeführt. Die Analysen zeigen, dass das umfangreiche Sparpaket des zweiten Tarifeingriffes die ambulante Versorgung (Einschränkung von Leistungen über Limitationen) und damit die Ziele der bundesrätlichen Strategie «Gesundheit 2020" einschränkt und erheblich gefährdet. Zudem werden die freie Praxis und die Spitalambulatorien durch den Eingriff teilweise ganz empfindlich finanziell getroffen.

Mit der nun vorliegenden definitiven Verordnung des Bundesrates werden die Massnahmen insgesamt zu einer deutlichen Schwächung der ambulanten Medizin führen, setzen keine Anreize für die Erbringung der Leistungen im deutlich günstigeren und effizienteren ambulanten Bereich und gefährden damit die jetzt schon problematische ambulante Versorgung der Bevölkerung in der Peripherie, ausserhalb der Ballungszentren.

\section{Auswirkungen auf den UVG-Bereich}

Die Verordnung des Bundesrates betrifft explizit nur die soziale Krankenversicherung KVG. Die MTK hat am 18. September 2017 entschieden, den bisherigen Tarif TARMED 01.08.00_BR bis zum 31. März 2018 unverändert gültig zu belassen und erst ab 1. April 2018 den veränderten Tarif 01.09.00_BR mit zusätzlichen UVG-Positionen sowie gewisse Leistungen mit einer höheren Abgeltung (z.B. Gutachten) in Kraft zu setzen. Die Details dazu liegen derzeit noch nicht vor. Dies bedeutet, dass mindestens für das erste Quartal 2018 zwei unterschiedliche ambulante TARMED-Tarifstrukturen parallel eingesetzt werden müssen (Unterscheidung nach angewendetem Gesetz KVG oder UVG). Auch dieser Umstand der gleichzeitigen Anwendung von 2 parallelen unterschiedlichen Tarifstrukturen, führt zu erheblichen Umsetzungsschwierigkeiten.

\section{Einzelne Massnahmen des zweiten Tarifeingriffes des Bundesrates in die Tarifstruktur TARMED per 01.01.2018}

Im Folgenden werden die wichtigsten Massnahmen des zweiten Tarifeingriffes des Bundesrates in die Tarifstruktur TARMED per 1.1.2018 kurz vorgestellt:

\section{Wichtig!}

Bitte Aktualisieren Sie Ihre Arztpraxissoftware mit der neuen Tarifstruktur TARMED Version 1.09.00_BR, sodass Sie ab dem 01.01.2018 Ihre Leistungen im Rahmen der sozialen Krankenversicherung KVG korrekt erfassen und abrechnen können. Ihr Software-Anbieter ist Ihnen dabei gerne behilflich. 


\section{Einheitlicher Dignitätsfaktor für alle Leistungen}

Die «Quantitativen Dignitäten" werden vereinheitlicht. Sämtlichen «Quantitativen Dignitäten» (FMHO5 bis FMH12) wird neu ein einheitlicher Dignitätsfaktor von 0.985 zugewiesen. Die Taxpunkte für die ärztliche Leistung mit der "Quantitativen Dignität» FMH5 (z.B. 00.0010 Konsultation, erste 5 Min. (Grundkonsultation) erhöhen sich (ursprünglicher Faktor = 0.905); die Taxpunkte der ärztlichen Leistung für alle Tarifpositionen mit einer höheren "Quantitativen Dignität» (FMHO6 bis FMH12) reduzieren sich auf den Dignitätsfaktor 0.985. Für den praktischen Arzt wird die ärztliche Leistung AL um den Skalierungsfaktor 0.93 reduziert (Basis neuer AL Taxpunkte).

\section{Erhöhung der ärztlichen Produktivität in den OP-Sparten (OP-Sparten)}

Die Produktivität in den OP-Sparten (OPI bis OPIII) wird angehoben. Die Produktivität der Sparte OP I erhöht sich von $45 \%$ auf 55\% (OPII von 50\% auf 60\% und OPIII von $55 \%$ auf $65 \%$ ). Dadurch reduzieren sich die Kostensätze der technischen Leistungen (TL). Die ärztliche Produktivität ist neben dem Referenzeinkommen, der Jahresarbeitszeit und der «Quantitativen Dignität» entscheidend für die Anzahl Taxpunkte der ärztlichen Leistung.

\section{Senkung der Kostensätze in gewissen Sparten}

Die Kostensätze von Sparten mit Investitionskosten für Anlagen, Geräte und Apparate über CHF 750000 werden um 10\% abgesenkt. Diese Massnahme betrifft 25 Sparten wie zum Beispiel die Sparten Praxis-OP und OP I. Von dieser Massnahme ausgeschlossen sind die Sparten-Kostensätze aller Untersuchungs- und Behandlungsräume (UBR) wie zum Beispiel UBR Grundversorger, UBR Chirurgie und Kinderchirurgie, UBR Ophthalmologie, UBR ORL, UBR Urologie, UBR Dermatologie und UBR Angiologie sowie die Sparte Gastroenterologische Endoskopie gross.

\section{Anpassung Minutage für die Leistung im} engeren Sinn bei CT und MRI-Untersuchungen

Bei den Tarifpositionen für die CT- und MRI-Untersuchungen wird die ärztliche Leistung im engeren Sinne gestrichen. Dafür gibt es neu für die ärztlichen Leistungen im engeren Sinne eine separate Tarifposition für den Fall, dass der Facharzt für Radiologie bei der Durchführung einer CT- oder MRI-Untersuchung für eine gewisse Zeit anwesend sein muss. Diese Tarifposition ist als Handlungsleistung mit einer Leistung im engeren Sinne von 5 Minuten tarifiert. Zudem wird die
Simulieren Sie die Auswirkungen auf Ihr

\section{Leistungsspektrum!}

Was bedeuten der Tarifeingriff des Bundesrates und die Massnahmen für die eigene Praxis? Muss ich mit Einbussen rechnen? Diese und ähnliche Fragen erreichen das Departement Ambulante Versorgung und Tarife aktuell täglich.

Die Simulationen mit dem Tool Volumis Online unterstützen alle FMH-Mitglieder bei der Beantwortung dieser Fragen und ermöglichen jeder Ärztin / jedem Arzt, die Auswirkungen des Tarifeingriffs bezogen auf sein individuelles Leistungsspektrum zu simulieren.

Mehr Informationen finden Sie auf myFMH und unserer Webseite: www.fmh.ch $\rightarrow$ AmbulanteTarife $\rightarrow$ TARMEDTarif $\rightarrow$ TARMED Änderungen ab 1.1.2018

Minutage für die Berichterstellung auf bei CT- bzw. MRI-Untersuchungen auf 20 bzw. 25 Minuten vereinheitlicht.

\section{Senkung der Minutagen bei ausgewählten Tarifpositionen}

Bei folgenden Leistungen werden die Minutagen der Leistung im engeren Sinne und der Raumbelegungszeit gesenkt: Kataraktoperation, Glaskörperbiopsie (u/o Intravitreale Injektion), Belastungs- und HolterEKG, Koloskopie und Stereotaktische Radiotherapie.

\section{6. Änderung des Leistungstyps bei «Untersuchung durch den Facharzt» von Handlungs- in Zeitleistungen}

Bei den Tarifpositionen «Untersuchung durch den Facharzt ...» wird der Leistungstyp von Handlungs- in Zeitleistungen geändert. Diese Tarifpositionen werden zukünftig nach Zeitaufwand pro 5 Minuten abgerechnet. Dies betrifft 10 Tarifpositionen wie zum Beispiel: Die Tarifposition 00.0410 Kleine Untersuchung durch den Facharzt für Grundversorgung (15 Min.) wird ersetzt durch die Tarifposition 00.0415 Kleine Untersuchung durch den Facharzt für Grundversorgung, pro 5 Min. und die Tarifposition 00.0420 Umfassende Untersuchung durch den Facharzt für Grundversorgung (25 Min.) wird ersetzt durch die Tarifposition 00.0425 Umfassende Untersuchung durch den Facharzt für Grundversorgung, pro 5 Min.

\section{Anwendung von Abrechnungslimitationen} auch für elektronisch abrechnende Fachärzte Der Vermerk «Diese Limitierung entfällt für elektronisch abrechnende Fachärzte» wird bei aktuell 20 Tarifpositionen ersatzlos gelöscht. Diese Massnahme betrifft zum Beispiel 00.0020 + Konsultation, jede weiteren $5 \mathrm{Min}$. (Konsultationszuschlag), 00.0070 
+ Besuch, jede weiteren 5 Min. (Besuchszuschlag) oder 00.0120 + Telefonische Konsultation durch den Facharzt, jede weiteren $5 \mathrm{Min}$. Die zeitlichen Limitationen werden für Kinder $<6$ Jahren und Personen $>75$ Jahren verdoppelt. Bei Patienten mit erhöhtem Behandlungsbedarf werden die Limitationen ebenfalls verdoppelt. Für Kinder $<6$ Jahren und Personen $>75$ Jahren sowie die Patienten mit erhöhtem Behandlungsbedarf werden neu jeweils separate Positionen geführt. Die Gründe für den erhöhten Behandlungsbedarf eines Patienten müssen in der Patientenakte aufgeführt werden und der erhöhte Behandlungsbedarf eines Patienten ist gegenüber dem Versicherer zu begründen.

\section{Differenzierung bei der "Leistungen in Abwesenheit des Patienten"}

Die Tarifposition 00.0140 Ärztliche Leistung in Abwesenheit des Patienten (inkl. Aktenstudium), pro 5 Min. wird aufgehoben zugunsten folgender neuer Tarifpositionen:

- 00.0141 Aktenstudium in Abwesenheit des Patienten, pro $1 \mathrm{Min}$

- 00.0142 Erkundigungen bei Dritten in Abwesenheit des Patienten, pro 1 Min.

- 00.0143 Auskünfte an Angehörige oder andere Bezugspersonen des Patienten in Abwesenheit des $\mathrm{Pa}$ tienten, pro $1 \mathrm{Min}$

- 00.0144 Besprechungen mit Therapeuten und Betreuern des Patienten in Abwesenheit des Patienten, pro $1 \mathrm{Min}$.

- 00.0145 Überweisungen an Konsiliarärzte in Abwesenheit des Patienten, pro $1 \mathrm{Min}$.

- 00.0146 Ausstellen von Rezepten oder Verordnungen ausserhalb von Konsultation, Besuch und telefonischer Konsultation in Abwesenheit des Patienten, pro $1 \mathrm{Min}$.

- 00.0147 Diagnostische Leistung am Institut für Pathologie in Abwesenheit des Patienten, pro 1 Min.

- 00.0148 Tumorboard in Abwesenheit des Patienten bei Personen über 6 Jahren und unter 75 Jahren, pro 1 Min.

Die heute hinterlegte Limitation der Tarifposition wird halbiert. Limitationen für Kinder $<6$ Jahren und Personen $>75$ Jahren werden nicht halbiert. Bei Patienten mit erhöhtem Behandlungsbedarf werden die Limitationen ebenfalls verdoppelt. Für Kinder $<6$ Jahren und Personen $>75$ Jahren sowie die Patienten mit erhöhtem Behandlungsbedarf werden neu jeweils separate Positionen geführt. Die Gründe für den erhöhten Behandlungsbedarf eines Patienten müssen in der Patientenakte aufgeführt werden und der erhöhte Behandlungsbedarf eines Patienten ist gegenüber dem Versicherer zu begründen.
Nebst den bereits jetzt aufgeführten Leistungen in der Interpretation der Tarifposition 00.0140 wird auch eine Position "Tumorboard in Abwesenheit des Patienten" für die Teilnahme an Tumorboards geschaffen. Die gleiche Ausdifferenzierung gilt auch für die Tarifpositionen 02.0070 Ärztliche Leistung in Abwesenheit des Patienten (inkl. Aktenstudium) durch den Facharzt für Psychiatrie, pro 5 Min. und 02.0160 Leistung in Abwesenheit des Patienten durch behandelnden Psychologen/Psychotherapeuten, pro 5 Min.

\section{Zuschläge für Notfall-Inkonvenienz- pauschale}

Die Interpretation bei den Tarifpositionen mit der Bezeichnung «Notfall-Inkonvenienzpauschale» wird präzisiert. Die Notfallkriterien für Notfälle während des Tages werden schärfer umschrieben, so dass klarer wird, in welchen Fällen diese Notfall-Inkonvenienzpauschale abgerechnet werden kann. Die Notfall-Inkonvenienzpauschalen können sowohl vom Arzt als auch von ambulanten Einrichtungen nach Artikel 36a KVG abgerechnet werden.

Die bis anhin geltenden Notfallkriterien für die Notfall-Inkonvenienzpauschale A (Mo-Fr 7-19, Sa 7-12) werden um einen Punkt ergänzt. Neu als Notfall-Kriterium aufgenommen wird, dass bei einem direkten Arzt-Patientenkontakt eine Störung der vitalen Funktionen vorhanden, zu befürchten bzw. nicht auszuschliessen sein müssen. Die Position ist auch abrechenbar bei Patienten, bei denen eine akute Erkrankung, ein Trauma oder eine Vergiftung eine Organschädigung hervorrufen oder zu Folge haben können. Im Bereich der Psychiatrie ist von einem Notfall auszugehen bei Erregungszuständen, Selbst- und Fremdgefährdung, Bewusstseinsstörungen, kataton-stuporösen $\mathrm{Zu}$ ständen. In Fällen ohne direkten Arzt-Patientenkontakt (z.B. Telefon) gelten nach wie vor die bisherigen Notfallkriterien.

Bei den Notfall-Inkonvenienzpauschalen B und C (MoSo 19-22, Sa 12-19, So 7-19 und Mo-So 22-7) werden die Notfallkriterien so ergänzt, dass bei einem direkten Arzt-Patientenkontakt der Facharzt die sofortige Behandlung als medizinisch notwendig erachtet. In Fällen ohne direkten Arzt-Patientenkontakt (z.B. Telefon) gilt nach wie vor, dass die sofortige Behandlung medizinisch notwendig ist und/oder vom Patienten, Angehörigen oder Dritten als offensichtlich notwendig erachtet wird. Weiter wird das Zeitfenster für die Dringlichkeits-Inkonvenienzpauschale $\mathrm{F}$ bei dringlichen Konsultationen/Besuchen ausserhalb der regulären Sprechstundenzeiten, sowie Mo-Fr 19-22, Sa 12-19, sowie So 7-19 auf den Samstagvormittag 7-12 erweitert. 
FMH / Abteilung Ambulante Versorgung und Tarife Baslerstrasse 47

CH-4600 Olten

Tel. 0313591230

Fax 0313591238

tarife.ambulant[at]fmh.ch
10. Anpassungen der Interpretation bei Punktion und Blutentnahme durch nicht ärztliches Personal (00.0715 und 00.0716)

Bei den Tarifpositionen 00.0715 Punktion, venös, zwecks Blutentnahme, jede Lokalisation durch nichtärztliches Personal und 00.0716 Blutentnahme kapillär, jede Lokalisation durch nichtärztliches Personal wird die Limitierung gelöscht, wonach diese nur durch das ärztliche Praxislabor im Rahmen der Präsenzdiagnostik anwendbar ist. Die nichtärztliche Blutentnahme kann auch dann abgerechnet werden, wenn die Analyse in einem externen Labor und nicht in der Arztpraxis erfolgt.

\section{Instruktion von Selbstmessung etc.}

Die Tarifposition 00.0610 Instruktion von Selbstmessungen, Selbstbehandlungen durch den Facharzt, pro 5 Min. ist neu in der Sparte Sprechzimmer und nicht mehr in der Sparte UBR Grundversorger tarifiert.

\section{Elimination des Zuschlags von $10 \%$ auf Verbrauchsmaterialien und Implantaten}

Der Zuschlag von 10\% auf den Einstandspreis von Verbrauchsmaterialien und Implantaten gemäß der Generellen Interpretation GI-20 Verbrauchsmaterialien und Implantate wird gestrichen.

\section{Zusätzliche Änderungen}

Die Tarifposition 00.2205 Formalisierter Arztbericht wird von einer Handlungsleistung in eine Zeitleistung mit Limitation (maximal 2 Mal pro Sitzung) umgewandelt und neu nummeriert (00.2206).

Es wird eine neue Tarifposition 02.0015 + Wegentschädigung für den Facharzt für Psychiatrie und Psychotherapie, pro 5 Min. eingefügt. Tarifiert ist die Position gleich wie die Psychiatrische Diagnostik und Therapie. Die Phytotherapie wird gleich tarifiert wie die andere Komplementärmedizin.

\section{Die FMH unterstützt Sie bei den Vorbereitungen}

Weitere Informationen zum zweiten Tarifeingriff des Bundesrates in die Tarifstruktur TARMED per 1.1.2018 finden Sie auf der Internetseite der FMH unter www. fmh.ch $\rightarrow$ TARMED $\rightarrow$ TARMED Änderungen $a b$ 1.1.2018. Auf der Internetseite der FMH werden unter anderem die Verordnung und die dazugehörige Unterlagen, ein Tarifbrowser inkl. Anleitung und Datenbank sowie diverse weitere Publikationen wie zum Beispiel FAQ und Fact-Sheet aufgeschaltet.

Die FMH bleibt auch weiterhin bei ihrer Position, dass nur eine umfassende Gesamtrevision des TARMED, gemeinsam mit allen Ärzteorganisationen und Tarifpartnern, der richtige Weg ist, damit eine sachgerechte und betriebswirtschaftliche Tarifstruktur wieder hergestellt werden kann, und verfolgt das Projekt TARCO deshalb mit grossem Einsatz weiter. 